\title{
Disponibilidade energética para a cultura da cana-de-açúcar nos tabuleiros costeiros do estado da Paraíba
}

\author{
Pedro V. de Azevedo ${ }^{1}$, Luciano M. F. Saboya ${ }^{2}$, José Dantas Neto ${ }^{3}$, \\ Fabricio da S. Oliveira ${ }^{4}$, José R. C. Bezerra ${ }^{5}$ \& Carlos H. de A. Farias ${ }^{6}$ \\ ${ }^{1}$ UACA/UFCG, Campina Grande, PB. E-mail: pvieira@dca.ufcg.edu.br \\ ${ }^{2}$ UAEA/UFCG, Campina Grande, PB. E-mail: saboya@deag.ufcg.edu.br (Autor correspondente) \\ ${ }^{3}$ UAEA/UFCG, Campina Grande, PB. E-mail: zedantas1955@gmail.com \\ ${ }^{4}$ UAEA/UFCG, Campina Grande, PB. E-mail: fabricio_silva51@hotmail.com \\ ${ }^{5}$ Embrapa Algodão, Campina Grande, PB. E-mail: jose.cortez-bezerra@embrapa.br \\ ${ }^{6}$ Miriri Alimentos e Bioenergia S/A, Santa Rita, PB.
}

\section{Palavras-chave:}

albedo

saldo de energia

fluxos de calor latente e sensível

fluxo de calor no solo

\begin{abstract}
R E S U M O
Um experimento foi conduzido com a cultivar de cana-de-açúcar (Saccharum spp.) RB92579, no período de 2010 a 2011 em Capim, PB, tendo como objetivo a análise dos componentes dos balanços de radiação e energia. Monitoraram-se as variáveis: radiação solar incidente e refletida; saldo de radiação; radiação atmosférica incidente à superfície, radiação efetiva emitida e o albedo da superfície vegetada. O balanço de energia, fundamentado na razão de Bowen, foi utilizado na determinação dos fluxos de calor latente e sensível para o ar e o solo. Para as fases de crescimento máximo e maturação observou-se que: a) não houve mudanças significativas no comportamento dos componentes do balanço de radiação; b) o albedo variou de 0,17 a 0,27 com média de 0,23 ; c) as regressões lineares entre o saldo de radiação e o saldo de radiação de ondas curtas apresentaram elevados níveis de correlação; d) o coeficiente térmico diminuiu e o coeficiente de transformação em ondas longas aumentou com o aumento da cobertura vegetal; e) o saldo de radiação diário e os fluxos de calor latente e sensível aumentaram do período chuvoso para o período seco e f) $82 \%$ do saldo de radiação foram utilizados como calor latente, $16 \%$ como calor sensível para aquecimento do ar e $2 \%$ como calor sensível para aquecimento do solo.
\end{abstract}

Key words:

albedo

net energy

fluxes of latent and sensible heat soil heat flux

\section{Availability of energy for sugarcane in the coastal plains of Paraíba state}

\begin{abstract}
A B S T R A C T
An experiment was conducted with the sugarcane (Saccharum spp.) variety RB92579, during the period of 2010 to 2011 in Capim,PB, aiming to analyse the components of the radiation and energy balances. The following variables were monitored: incident and reflected solar radiation, net radiation, atmospheric radiation incident to the surface, emitted effective radiation and the albedo of the vegetated surface. The energy balance based on the Bowen ratio was used to determine the latent and air and soil sensible heat fluxes. For the sugarcane maximum growth and maturation phases showed that: a) there were no significant changes in the behavior of the radiation balance components, b) the albedo ranged from 0.17 to 0.27 with an average of 0.23 ; c) the linear regressions between net radiation and net shortwave radiation showed high levels of correlation; d) the thermal coefficient decreased while the coefficient of transformation into long waves increased with increasing vegetation cover, e) the daily net radiation and the latent and sensible heat fluxes increased from the rainy to the dry seasons; f) $82 \%$ of net radiation was used as latent heat, $16 \%$ and $2 \%$ as sensible heat for heating air and the soil, respectively.
\end{abstract}

\section{INTRODUÇÃO}

O Brasil é um dos maiores produtores de cana-de-açúcar no mundo, com rendimento médio de $76,4 \mathrm{t} \mathrm{ha}^{-1}$ na safra 2011 (IBGE, 2012). O estado de São Paulo é o principal produtor nacional, com $58 \%$ da produção de etanol e açúcar e $70 \%$ das exportações (Armas et al., 2005). O estado da Paraíba apresenta produtividade média de $54,34 \mathrm{t} \mathrm{ha}^{-1}$, situando-se na décima oitava posição dentre os vinte e um estados produtores do país (IBGE, 2012).
A produção agrícola é diretamente influenciada pelo clima, em especial pela temperatura do ar, precipitação pluvial e radiação solar. Os balanços de radiação e energia têm sido objeto de vários trabalhos de pesquisa que buscam explicar seu comportamento em cana-de-açúcar (André et al., 2010a; 2010b), em mangueira (Borges et al., 2008) e em áreas irrigadas ou de caatinga do semiárido (Silva et al., 2005).

Os processos radiativos que interagem no sistema soloplanta-atmosfera podem ser avaliados por meio de medições micrometeorológicas (Qin et al., 2008) possibilitando conhecer 
as frações da energia disponível destinadas ao aquecimento do ar, solo e a utilizada no processo de transferência de vapor de água para a atmosfera (Shen et al., 2004). Esta partição fornece informações a respeito da evapotranspiração e do coeficiente de cultura (Kjaersgaard et al., 2008).

Para a partição da energia disponível entre os fluxos de calor latente e sensível o método mais utilizado é o do balanço de energia (Azevedo et al., 2003, 2006; Righi et al., 2007; Teixeira et al., 2008) tendo, como vantagens, a simplicidade, o baixo custo e o fato de apresentar bons resultados quando comparados aos métodos dos vórtices turbulentos e lisimétricos (Silva et al., 2005; Gavilán \& Berengena, 2007) além de desempenho superior ao método aerodinâmico (Cabral et al., 2003). Contudo, o cálculo dos fluxos pelo método do balanço de energia depende da determinação correta da razão de Bowen, sendo afetada pela precisão e exatidão das medições de temperatura e umidade do ar acima da cultura (Perez et al., 1999; Oliveira \& Leitão, 2000; Inman-Bamber \& Mcglinchey, 2003).

Esta pesquisa objetivou a avaliação da disponibilidade energética para cana-de-açúcar nos Tabuleiros Costeiros do estado da Paraíba.

\section{Material e Métodos}

O experimento de campo foi conduzido na Miriri Alimentos e Bioenergia S/A (6 $54^{\prime} 59^{\prime \prime}$ S, $35^{\circ} 09^{\prime} 17^{\prime \prime}$ W e $121 \mathrm{~m}$ de altitude), no município de Capim, PB, no terceiro ciclo de produção (2010/2011) da cana-de-açúcar (Saccharum spp.), cultivar RB92579, com espaçamento de 1,0 m entre linhas, em condições de sequeiro. Segundo classificação de Köppen, o clima é classificado como As' (quente e úmido), a temperatura e a precipitação média anual correspondem a $28^{\circ} \mathrm{C}$ e $1.600 \mathrm{~mm}$ (Silva, 2004). O solo da área experimental foi classificado como Argissolo vermelho amarelo com textura franco-argilo-arenosa.

Em uma parcela experimental de 25 ha foi instalada uma torre micro-meteorológica com os seguintes sensores: dois piranômetros (CM3, Kipp \& Zonen, Delft, Holanda), para medição da radiação solar global incidente (Rsi) e refletida (Rsr); um saldo radiômetro (NR LITE, Kipp \& Zonen, Delft, Holanda) para medição do saldo de radiação (Sr); dois psicrômetros aspirados com termopares de cobre e constantan (Tipo T, Omega, Stamford, CT, USA) instalados em dois níveis mantidos a 0,30 e 1,50 m acima da vegetação, para medição das temperaturas do ar em bulbos seco e úmido; dois anemômetros (Gill3 cup - modelo 12102, Young, Traverse City, Michigan, USA) para medição da velocidade do vento, em dois níveis e nas mesmas alturas dos sensores de temperatura; dois fluxímetros (Modelo HFT3, da Campbell Scientific Inc., Logan, Utah, USA) instalados a $0,08 \mathrm{~m}$ de profundidade para medir o fluxo de calor no solo, sendo um entre fileiras e outro na fileira de plantas. Todos os sensores foram conectados a um sistema automático de aquisição de dados (Datalogger - modelo CR1000, Campbell Scientific Inc., Logan, Utah, USA) programado para efetuar leituras dos sinais analógicos e digitais a cada $5 \mathrm{~s}$ e extrair e armazenar as médias para intervalos de $20 \mathrm{~min}$.
O balanço de radiação sobre a vegetação foi obtido como:

$$
\mathrm{Sr}=\mathrm{Rsi}-\mathrm{Rsr}+\mathrm{Rli}-\mathrm{Rle}-(1-\varepsilon) \mathrm{Rli}
$$

onde: Sr é o saldo de radiação; Rsi e Rsr se referem à radiação solar incidente e refletida, respectivamente; Rli, à radiação atmosférica incidente à superfície; Rle a emissão efetiva da superfície e $\varepsilon$ a emissividade da superfície, todos em $\mathrm{W} \mathrm{m}^{-2}$.

Também foram analisados os parâmetros derivados do balanço de radiação (André et al., 2010a):

a) Albedo ou coeficiente de reflexão da superfície:

$\mathrm{O}$ albedo da superfície vegetativa foi obtido por:

$$
\alpha=\frac{\mathrm{Rsr}}{\mathrm{Rsi}}
$$

b) Relação entre o saldo de radiação e o balanço de radiação de ondas curtas

Os coeficientes angular (a) e linear (b) da regressão entre o saldo de radiação $\left(\mathrm{S}_{\mathrm{r}}\right)$ e a diferença (Rsi - Rsr) ou $(1-\alpha)$ Rsi, foram obtidos como:

$$
\mathrm{Sr}=\mathrm{a}+\mathrm{b}(1-\alpha) \operatorname{Rsi}
$$

As propriedades térmicas do ar $(\tau \mathrm{e} \lambda)$ foram obtidas como:

$$
\begin{gathered}
\tau=\frac{1-\mathrm{a}}{\mathrm{a}} \\
\lambda=\mathrm{a}-1
\end{gathered}
$$

onde:

$\tau$ - coeficiente térmico

$\lambda$ - coeficiente de transformação em ondas longas

c) Transmissividade atmosférica

A transmissividade radiativa da atmosfera (Tr) correspondente à nebulosidade, foi calculada por:

$$
\operatorname{Tr}=\frac{R s i}{\operatorname{Ra}}
$$

Os limites utilizados para transmissividade da atmosfera foram $(\operatorname{Tr}<0,20 \rightarrow$ nublado; $0,20<\operatorname{Tr}<0,80 \rightarrow$ parcialmente nublado e $\operatorname{Tr}>0,80 \rightarrow$ céu claro). A radiação solar global no topo da atmosfera $(\mathrm{Ra})$ foi obtida em função da latitude do local, da declinação solar e do ângulo horário (Vianello \& Alves, 1991):

$$
\mathrm{Ra}=\mathrm{So}\left(\frac{\mathrm{D}^{\prime}}{\mathrm{D}}\right)^{2} \cos \mathrm{Z}
$$

onde:

$\mathrm{S}_{0} \quad$ - constante solar $\left(\mathrm{S}_{0}=1.367 \mathrm{~W} \mathrm{~m}^{-2}\right)$

D - distância Terra-Sol para determinado dia do ano 
D’ - distância média Terra-Sol e Z o ângulo zenital, sendo $\left(\mathrm{D}^{\prime} / \mathrm{D}\right)^{2}$ o fator de correção devido à excentricidade da órbita da terra.

O saldo de radiação de ondas longas ( $\mathrm{Srl}$ ) foi obtido por diferença entre o saldo de radiação $\left(\mathrm{S}_{\mathrm{r}}\right)$ e o saldo de radiação ondas curtas por:

$$
\mathrm{Srl}=\mathrm{Sr}-(1-\alpha) \mathrm{Rsi}
$$

As fases fenológicas consideradas para a cana-de-açúcar foram: I - Perfilhamento, do início até 110 dias após o corte (DAC); II - crescimento dos colmos (111 $\leq$ DAC $\leq 261)$; III - crescimento máximo dos colmos $(262 \leq \mathrm{DAC} \leq 347)$ e IV maturação dos colmos ( $348 \leq \mathrm{DAC} \leq 383)$. Entretanto, serão apresentados apenas os valores médios dos totais diários dos diversos componentes do balanço de radiação para as fases fenológicas III e IV. Todos os parâmetros derivados do balanço de radiação foram estimados para o período diurno, quando $(\mathrm{Rn}-\mathrm{G})>0$. Para cada fase fenológica os resultados também foram analisados como a média de dias nublados, parcialmente nublados e de céu claro.

Os componentes do balanço de energia na vegetação foram obtidos pelo método do balanço de energia baseado na razão de Bowen (BERB), pela Eq. 9 (Perez et al.,1999):

$$
\mathrm{Sr}+\mathrm{LE}+\mathrm{H}+\mathrm{G}=0
$$

onde:

$\mathrm{Sr}$ - saldo de radiação

LE - fluxo de calor latente

$\mathrm{H}$ e $\mathrm{G}$ - fluxos de calor sensível para o ar e para o solo, respectivamente, todos em $\mathrm{W} \mathrm{m} \mathrm{m}^{-2}$

Neste processo as densidades de fluxo que chegam à camada vegetativa são positivas enquanto as que saem são negativas.

A razão de Bowen ( $\beta=\mathrm{H} / \mathrm{LE}$ ) foi estimada de acordo com a Eq. 10 (Perez et al., 2008) considerando $\mathrm{Kh} \cong \mathrm{Kw}$ :

$$
\beta=\frac{\mathrm{H}}{\mathrm{L}_{\mathrm{E}}}=\frac{\mathrm{Po} \cdot \mathrm{Cp}}{\mathrm{L}_{\mathrm{E}}}\left(\frac{\mathrm{Kh}}{\mathrm{Kw}}\right) \frac{\frac{\partial \mathrm{T}}{\partial \mathrm{Z}}}{\frac{\partial \mathrm{e}}{\partial \mathrm{Z}}}=\gamma\left(\frac{\mathrm{Kh}}{\mathrm{Kw}}\right) \frac{\Delta \mathrm{T}}{\Delta \mathrm{e}}
$$

em que:

Kh e Kw - coeficientes de difusão turbulenta de calor sensível e vapor d'água, respectivamente, $\mathrm{m}^{2} \mathrm{~s}^{-1}$

L - o calor latente de evaporação da água, $\mathrm{MJ} \mathrm{kg}^{-1}$

$\mathrm{Cp}$ - o calor específico do ar secado a pressão constante, $\mathrm{MJ} \mathrm{kg-1}{ }^{\circ} \mathrm{C}^{-1}$

Po - a pressão atmosférica média local, $\mathrm{kPa}$

$\varepsilon=0,622$ - razão entre as massas moleculares da água e do ar seco $\Delta \mathrm{T}=\mathrm{T}_{2}-\mathrm{T}_{1}$ e $\Delta \mathrm{e}=\mathrm{e}_{2}-\mathrm{e}_{1}$ - as diferenças de temperatura do ar e da pressão parcial do vapor d'água atmosférico, medidas em dois níveis acima da vegetação $\left(\Delta z=z_{2}-z_{1}\right)$

$\gamma=(\mathrm{CpPo}) /\left(\mathrm{L}_{\mathrm{E}}\right)$ o fator psicrométrico $\left(\mathrm{kPa}^{\circ} \mathrm{C}^{-1}\right)$
Na ausência de advecção de calor sensível e umidade e em condições de neutralidade atmosférica, $\mathrm{Kh} \cong \mathrm{Kw}$ considerando que $[(\partial \mathrm{T} / \partial \mathrm{Z}) /(\partial \mathrm{e} / \partial \mathrm{Z})] \cong \Delta \mathrm{T} / \Delta \mathrm{e}$, a razão de Bowen pode ser obtida por:

$$
\beta=\gamma\left(\frac{\Delta \mathrm{T}}{\Delta \mathrm{e}}\right)=\left(\frac{\Delta+\gamma\left(\mathrm{T}_{\mathrm{bu} 1}-\mathrm{T}_{\mathrm{bu} 2}\right)}{\gamma\left(\mathrm{T}_{\mathrm{bu} 1}-\mathrm{T}_{\mathrm{bu} 2}\right)}-1\right)^{-1}
$$

em que:

$\Delta \quad$ - declividade da curva de pressão de saturação de vapor d'água, $\mathrm{kPa}{ }^{\circ} \mathrm{C}^{-1}$

$\mathrm{T}_{\text {bu1 }}$ e $\mathrm{T}_{\mathrm{bu} 2}$ - temperatura de bulbo molhado em dois níveis de medida acima do dossel da cultura, ${ }^{\circ} \mathrm{C}$

$\mathrm{T}_{\mathrm{bs} 1}$ e $\mathrm{T}_{\mathrm{bs} 2}$ - temperatura de bulbo seco em dois níveis de medida acima do dossel da cultura, ${ }^{\circ} \mathrm{C}$

Visando eliminar alguns erros associados ao método BERB aplicado às estimativas dos valores de $\beta$ e consequente cálculo do fluxo LE e $\mathrm{H}$, foram considerandas as situações em que (Rn $-\mathrm{G})>0$ e $\beta>-1$, quando o valor de $\mathrm{LE}>0$ e $\mathrm{H}$ podendo ser positivo $(\beta>0)$ ou negativo $(-1<\beta \leq 0)$ (Perez et al., 1999; Silva et al., 2011). Adicionalmente, considerou-se que valores de $\beta<$ - 0,75 resultam em valores de LE e H fisicamente inconsistentes (Ortega-Farias et al., 1996).

O fluxo de calor latente $\left(\mathrm{L}_{\mathrm{E}}\right)$ foi obtido como:

$$
\mathrm{LE}=-\left(\frac{\mathrm{Sr}+\mathrm{G}}{1+\beta}\right)
$$

\section{Resultados e Discussão}

Observou-se que os dados analisados apresentaram consistência superior a 90\%, segundo os critérios adotados. Comportamento similar foi observado por Silva et al. (2011) quando analisaram o período diurno de coleta de dados no cultivo de cana-de-açúcar, em Juazeiro, BA.

Como esperado, o albedo apresentou valores mais elevados nas primeiras horas da manhã e ao entardecer e menores valores em torno do meio-dia confirmando a dependência do albedo em função do ângulo de elevação solar. O valor mínimo por volta do meio-dia é atribuído ao menor ângulo de incidência dos raios solares causando maior penetração e retenção da radiação no interior da comunidade vegetal.

O albedo diário apresentou valores máximos $(27,4 \%)$ e mínimos $(17,3 \%)$ no primeiro mês de observações (junho); depois, voltou a decrescer durante os meses de agosto e setembro variando de 29 a $23 \%$ ao final do estágio de maturação dos colmos. O valor médio para o estádio de desenvolvimento de colmos variou entre 19 e 30\%, para o estádio de maturação obteve-se $27 \%$ e a média para todo o período de observação foi de 22,85\%. Este comportamento ocorreu em função da frequência de chuvas no período. Em junho ocorreram 17 dias chuvosos totalizando 106,8 mm de precipitação; em julho 21 dias e $330,3 \mathrm{~mm}$, em agosto 13 dias e $72,5 \mathrm{~mm}$ e em setembro 
3 dias e $16 \mathrm{~mm}$ de precipitação, o que proporcionaria oscilação da quantidade de radiação refletida alterando o valor do albedo.

André et al. (2010a) encontraram albedo crescente com o aumento da área foliar da cana-de-açúcar com valores médios de $0,24,0,27,0,30$ e 0,31 para os estádios de perfilhamento, desenvolvimento inicial de colmos, desenvolvimento de colmos e maturação, respectivamente, além de valor médio para todo o ciclo da cultura, de 0,28 $\pm 0,029$. Silva et al. (2011) obtiveram, para o ciclo da cana-de-açúcar, albedo médio de $23 \pm 3 \%$, com variações entre 15 e 27\%. Esteves et al. (2011) obtiveram, trabalhando com cana-de-açúcar em Campos de Goytacazes, RJ, albedo médio de 0,23 para o perfilhamento, de 0,28 para o desenvolvimento dos colmos e 0,26 para a maturação, para todo o ciclo de 0,26.

Em geral, as regressões lineares entre $\mathrm{Sr}$ e $(1-\alpha) \cdot \mathrm{R}_{\mathrm{si}}$, se ajustaram bem aos dados para todos os meses considerados (Tabela 1).

A transmissividade atmosférica variou de 0,11 a 0,50 , com média no período de 0,30 , significando que, na maioria dos dias, predominaram condições de céu parcialmente nublado.

$\mathrm{O}$ coeficiente térmico $(\tau)$ diminui com o aumento da cobertura vegetal ocorrendo o contrário com o coeficiente de transformação em ondas longas $(\lambda)$ variando de $-0,68$ a $-0,26$ e de 0,35 a 2,11, respectivamente (Tabela 1 ). Esses coeficientes são ferramentas úteis no entendimento das transformações de energia na superfície e na atmosfera por terem apresentado diferenças sensíveis nos diversos estádios fenológicos da cultura da cana-de-açúcar (André et al., 2010a).
Fisicamente, $\tau$ representa a parcela do saldo de radiação convertido em ondas longas; assim, um aumento na radiação solar global implica em aumento na emissão efetiva da superfície, o que provoca um aumento em $\tau$. Os menores valores de $\tau$ (maiores valores de $\lambda$ ), no estádio de desenvolvimento vegetativo indicaram que a maior parcela do saldo de radiação está sendo convertida em calor latente.

Desta forma, as magnitudes dos termos do balanço de radiação e dos parâmetros derivados, ao longo do ciclo de desenvolvimento da cana-de-açúcar são usadas para os cálculos do balanço de energia e do fluxo de vapor d'água na segunda parte deste estudo.

A análise da Figura 1 evidencia que, para o estádio de desenvolvimento máximo dos colmos (262 a 347 DAC), o saldo de radiação $(\mathrm{Sr})$ esteve em consonância com a radiação solar incidente (Rsi) e refletida (Rsr).

Para o estádio de maturação dos colmos (348 a 383 DAC) o Sr seguiu o comportamento de Rsi e Rsr. O Srl apresentou valores bem pequenos e às vezes negativos; aos $364 \mathrm{DAC}, \mathrm{T}_{\mathrm{r}}$ foi de 0,34 , sendo que o dia selecionado para caracterizar o estádio de maturação apresentou os seguintes valores das variáveis atmosféricas: temperatura máxima de $31,2{ }^{\circ} \mathrm{C}$, Rsi de $12,23 \mathrm{MJ}$ $\mathrm{m}^{-2}, \mathrm{Sr}$ de $9,45 \mathrm{MJ} \mathrm{m}^{-2}$. Neste período as chuvas já tinham cessado e a cultura se encontrava em fase de maturação; portanto, a absorção de energia seria menor.

A variação média dos totais diurnos do $\mathrm{Sr}(\mathrm{A}), \mathrm{LE}(\mathrm{B}), \mathrm{H}$ (C) e G (D) está apresentada nas Figuras 2 e 3.

Tabela 1. Parâmetros derivados do balanço de radiação para as fases fenológicas: III - crescimento máximo dos colmos (262 a 347 DAC) e IV - maturação dos colmos (348 a 383 DAC) da cultura da cana-de-açúcar nos Tabuleiros Costeiros do estado da Paraíba, ciclo de 2010/2011

\begin{tabular}{|c|c|c|c|c|}
\hline \multirow{2}{*}{ Parâmetros do balanço de radiação } & \multicolumn{4}{|c|}{ Mês/Fase fenológica } \\
\hline & Jun/III & Jul/III & Ago/III & Set/IV \\
\hline Coeficiente angular (a) & 2,17 & 2,64 & 3,110 & 3,710 \\
\hline Coeficiente linear (b) & 2,57 & 1,57 & 0,094 & $-0,095$ \\
\hline Coeficiente de determinação $\left(r^{2}\right)$ & 0,42 & 0,65 & 0,860 & 0,950 \\
\hline Transmissividade atmosférica $\left(\mathrm{T}_{\mathrm{r}}\right)$ & 0,30 & 0,30 & 0,310 & 0,320 \\
\hline Coeficiente térmico $(\tau)$ & $-0,54$ & $-0,62$ & $-0,680$ & $-0,260$ \\
\hline Coeficiente de transformação em ondas longas $(\lambda)$ & 1,17 & 1,64 & 2,110 & 0,350 \\
\hline
\end{tabular}

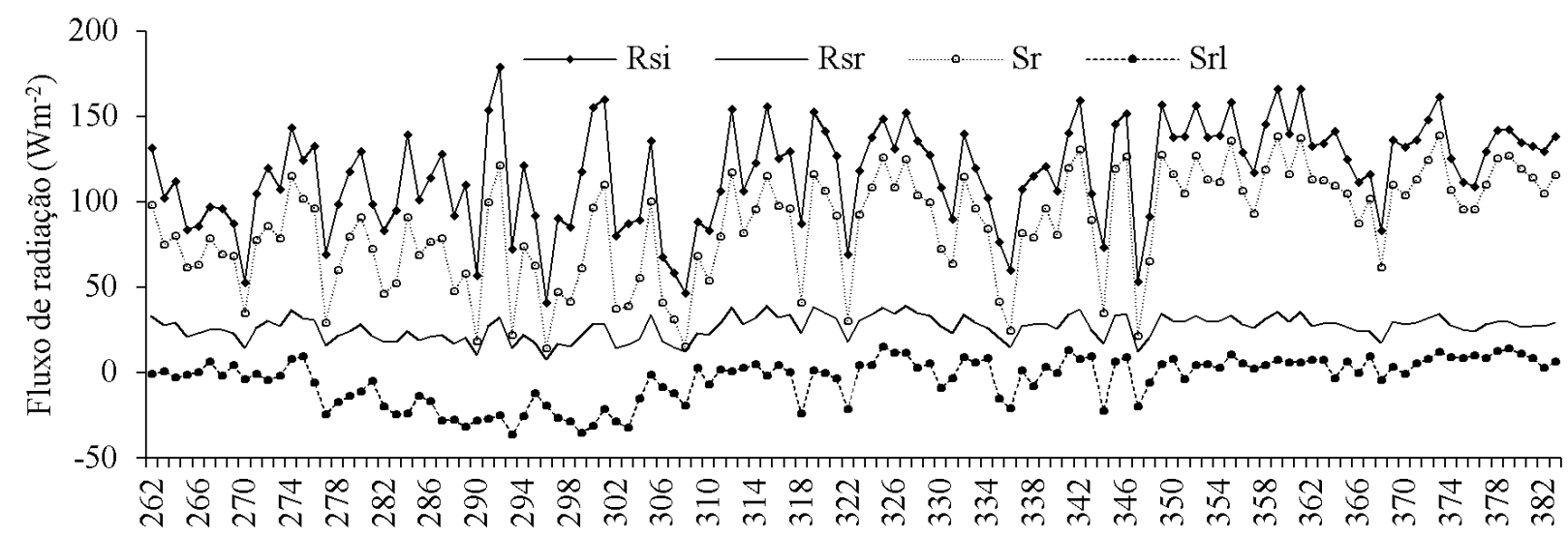

Dias após o corte

Figura 1. Totais diários dos termos do balanço de radiação para as fases fenológicas III - crescimento máximo dos colmos (262 a 347 DAC) e IV - maturação dos colmos (348 a 383 DAC) da cultura da cana-de-açúcar nos Tabuleiros Costeiros do estado da Paraíba, ciclo de 2010/2011 
A.

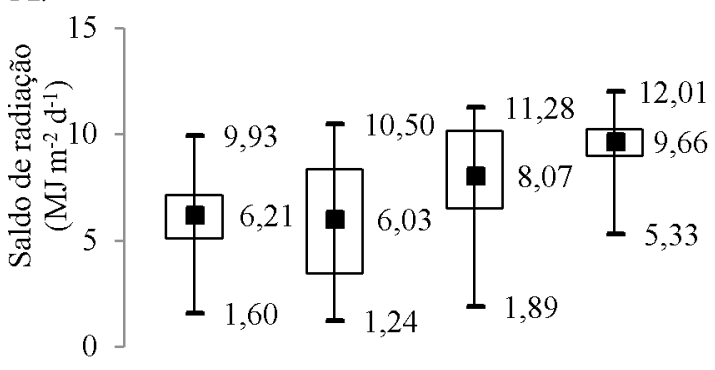

C.

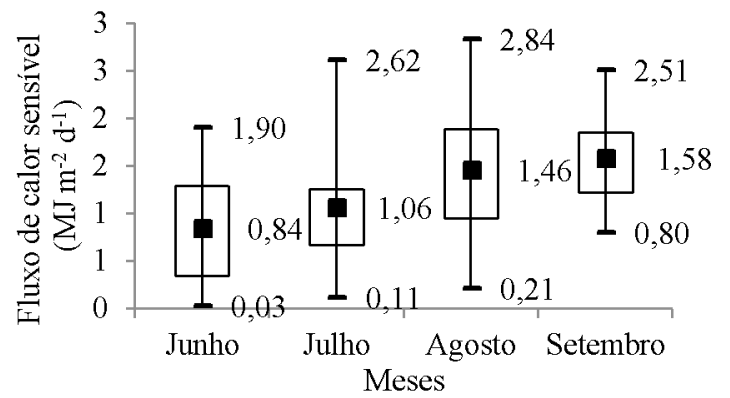

B.

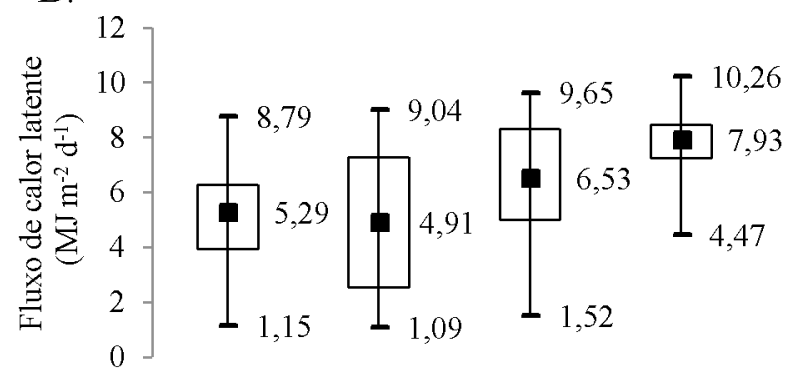

D.

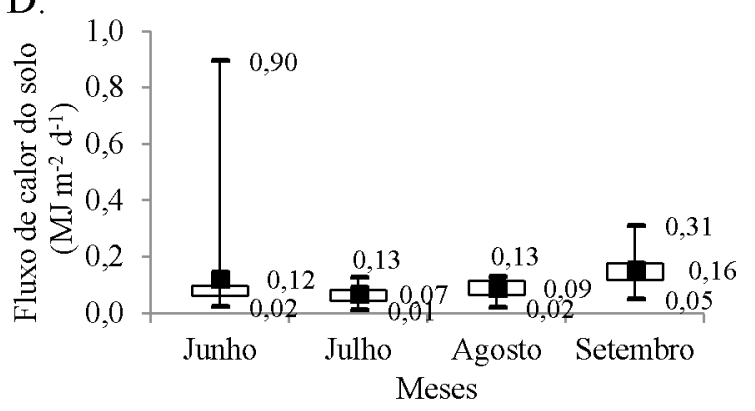

Figura 2. Valores máximos, mínimos e médios dos totais diurnos dos termos do balanço de energia para os meses de junho, julho, agosto e setembro, na cultura da cana-de-açúcar, nos Tabuleiros Costeiros do estado da Paraíba, ciclo de 2010/2011

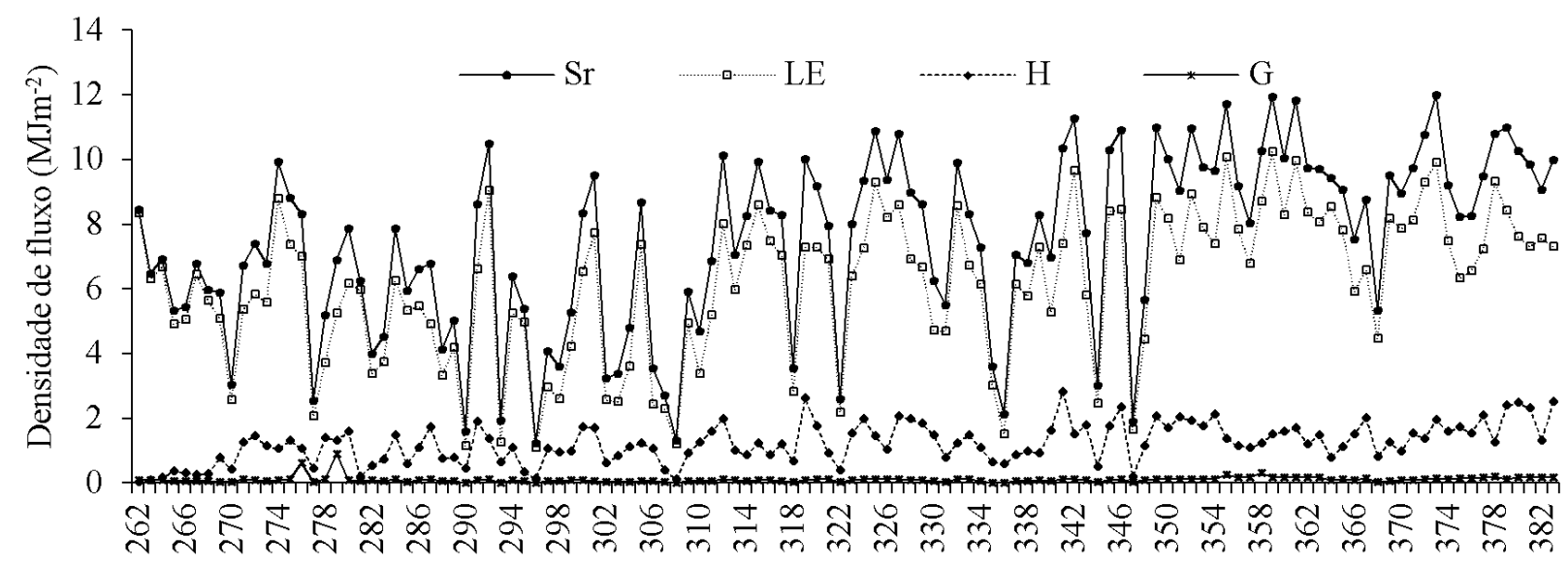

Dias após o corte

Figura 3. Comportamento do saldo de radiação (Sr), o fluxo de calor latente (LE), fluxo de calor sernsível para o ar (H) e para o solo (G) para as fases fenológicas III - crescimento máximo dos colmos (262 a 347 DAC) e IV - maturação dos colmos (348 a 383 DAC) da cultura da cana-de-açúcar nos Tabuleiros Costeiros do estado da Paraíba, ciclo de 2010/2011

O saldo de radiação apresentou oscilações diárias; mesmo assim, não houve mudanças significaticas ao longo do período estudado; por outro lado, as magnitudes dos fluxos médios diários de LE, $\mathrm{H}$ e G acompanharam aproximadamente esta tendência. Ao longo do período o LE não só apresentou grande variação mas também a maior parcela do saldo de radiação.

Não foram observadas mudanças significativas no comportamento dos termos do balanço de energia correspondentes às fases de desenvolvimento máximo dos colmos e maturação da cana-de-açúcar embora os valores absolutos dos termos tenham sido diferentes (Figura 2).

Os valores máximo, mínimos e médios mensais para os principais componentes da equação do balanço de energia são apresentados na Tabela 2.
Observando as médias dos referidos meses nota-se um aumento do saldo de radiação e dos fluxos de calor latente, sensível e do solo, em razão das alterações meteorológicas ocorridas no período. Analisando os dados de todo o período estudado constatou-se que o saldo de radiação variou entre 1,24 e 12,01 MJ m ${ }^{-2}$ com média de 7,49 $\mathrm{MJ} \mathrm{m}^{-2}$, o LE esteve na faixa de 1,09 a $10,26 \mathrm{MJ} \mathrm{m}^{-2}$ com média $6,16 \mathrm{MJ} \mathrm{m}^{-2}$, o H compreendeu entre 0,03 e $2,84 \mathrm{MJ} \mathrm{m}^{-2}$ com média de $1,24 \mathrm{MJ} \mathrm{m}^{-2}$ e G variou entre 0,01 e $0,90 \mathrm{MJ} \mathrm{m}^{-2}$ com média de $0,11 \mathrm{MJ} \mathrm{m}^{-2}$.

Verifica-se, em canaviais, que quando a cultura dispõe suficientemente de umidade tanto no solo como no dossel, a maior quantidade do Sr é utilizada na evapotranspiração, na forma de LE, e em menores quantidades são emitidas por $\mathrm{H}$ e $G$, mostrando que essa diferença ocorreu em virtude da alta 
Tabela 2. Valores médios dos componentes do balanço de energia* $(\mathrm{Sr}, \mathrm{LE}, \mathrm{H}, \mathrm{G})$ e da partição do Sr em fluxo de calor latente (LE/Sr), calor sensível do ar (H/Sr), fluxo de calor no solo (G/Sr) para as fases fenológicas III - crescimento máximo dos colmos (109 a 347 DAC) e IV - maturação dos colmos (348 a 383 DAC) da cultura da cana-de-açúcar, nos Tabuleiros Costeiros do estado da Paraíba, ciclo de 2010/2011

\begin{tabular}{|c|c|c|c|c|c|c|c|}
\hline \multirow{2}{*}{ Mês } & $\mathrm{Sr}$ & LE & $\mathrm{H}$ & G & $\mathrm{LE} / \mathrm{Sr}$ & $\mathrm{H} / \mathrm{Sr}$ & $\mathrm{G} / \mathrm{Sr}$ \\
\hline & \multicolumn{4}{|c|}{ MJ $\mathrm{m}^{-2}$} & \multicolumn{3}{|c|}{$\%$} \\
\hline Junho & 6,21 & 5,29 & 0,84 & 0,120 & 85,0 & 13,0 & 2,0 \\
\hline Julho & 6,03 & 4,91 & 1,06 & 0,007 & 81,1 & 17,6 & 1,3 \\
\hline Setembro & 9,66 & 7,93 & 1,58 & 0,160 & 82,1 & 16,3 & 1,6 \\
\hline Média & 7,49 & 6,16 & 1,24 & 0,110 & 82,3 & 16,2 & 1,5 \\
\hline
\end{tabular}

* Sr - saldo de radiação; LE - fluxo de calor latente; H - fluxo de calor sensível para 0 ar; $\mathrm{G}$ - fluxo de calor sensível para 0 solo

variação na nebulosidade e precipitações. Esses resultados concordam com os obtidos em vários trabalhos com balanço de energia em culturas anuais e perenes, através dos quais se concluiu que a maior porção do saldo de radiação é transformada em fluxo de calor latente (Teixeira, 2001; André et al., 2010b; Silva et al., 2011).

Estudando os aspectos energéticos do desenvolvimento da cana-de-açúcar, André et al. (2010b) obtiveram Sr variando de 10,9 a $17,26 \mathrm{MJ} \mathrm{m}^{-2} \mathrm{~d}^{-1}$, sendo a maior parte utilizada pelo LE que variou de $79 \%$ no início do perfilhamento a $99 \%$ no final da maturação; já o $\mathrm{H}$ apresentou pequenas variações com valores muito baixos tal como, também, o G. O excesso de umidade no solo ou no dossel da cana-de-açúcar, contribuiu durante o período estudado, para os elevados valores de LE uma vez que a energia, ao incidir sobre a superfície, é utilizada prioritariamente no processo de transferência de água, na forma de vapor, para a atmosfera (evapotranspiração) para, posteriormente, em menor quantidade ser utilizada nos processos de aquecimento do ar (H) e do solo (G).

Ao longo do período de observações o Sr diurno foi menor nos meses de junho e julho (período chuvoso) e maior no mês de setembro (período seco). Quanto aos demais componentes do balanço de energia o comportamento de LE, H e G seguiu aquele do Sr, com valores máximos em torno do meio-dia e mínimo (zero) ao nascer e pôr-do-sol.

As relações percentuais entre $\mathrm{LE}, \mathrm{He} \mathrm{Ge}_{\mathrm{r}}$ são apresentadas na Tabela 2 podendo-se verificar que $\mathrm{o} L E$ correspondeu à maior partição e que nos meses de junho e julho, mesmo ocorrendo decréscimo, ele se manteve estável até o mês de agosto para, posteriormente, haver um pequeno acréscimo em setembro.

$\mathrm{H}$ e $\mathrm{G}$ apresentaram valores muito baixos da partição da radiação líquida proporcionando, também, pequenas oscilações durante os meses. O decréscimo e o acréscimo ocorridos nos referidos meses não proporcionam grandes diferenças em comparação individual das partições da radiação líquida, o que pode estar associado ao período que compreendeu a pesquisa. Esta ordem de grandeza, com predominância de Sr para LE, seguido de $\mathrm{He}$ G, tem sido padrão observado nas determinações do balanço de energia, que empregam o uso da razão de Bowen em culturas agrícolas (Silva et al., 2009; André et al., 2010b).

No período de observações 82,$3 ; 16,2$ e 1,5\% do Sr foram consumidos como fluxos LE, H e G, respectivamente (Tabela 2). Em cultivo da cana-de-açúcar no estado do Rio de Janeiro, André et al. (2010b) observaram que para os dias típicos o fluxo de vapor d'água representou 78\% $(\mathrm{DAC}=109), 80 \%(\mathrm{DAC}=$
197), $91 \%(\mathrm{DAC}=260)$ e $96 \%(\mathrm{DAC}=339)$ do valor do $\mathrm{S}_{\mathrm{r}}$ e constataram também, para as fases de perfilhamento, que no desenvolvimento de colmos e maturação o fluxo de vapor d'água apresentou valores médios de 79; 87; 99 e 120\% do Sr, respectivamente.

A fração do Sr utilizado como LE variou de $85 \%$ em junho a $82,1 \%$ em setembro. A participação do H variou de $13 \%$ em junho ao valor máximo de $18 \%$ em agosto; já a partição do $G$ variou de 2,0\% em junho, sendo o valor máximo, a 1,6\% em setembro.

Conforme as razões de partição do Sr para LE, H e G (Tabela 2) constatou-se que, em média, $\mathrm{Sr}$ foi consumido em $82,3 \%$ para LE, $16,2 \%$ como H e 1,5\% como G, considerandose todos os meses estudados. André et al. (2010b) obtiveram valores superiores para os dias analisados durante a fase de desenvolvimento dos colmos e consumo de $86 \%$ do Sr pelo LE, em cultivo que não sofreu estresse hídrico.

Silva et al. (2011) obtiveram resultados que se assemelham em partes aos desta pesquisa verificando que, em média, a maior porção do Sr disponível para o cultivo foi utilizada para o LE (81\%) seguido do H (16\%) e o G (3\%). Já Silva et al. (2009) obtiveram resultados semelhante aos desta pesquisa, com $78 \%$ do $\mathrm{Sr}$ sendo utilizado como LE, $18 \%$ como $\mathrm{H}$ e $4,4 \%$ como G. Os autores ainda observaram que a prática de irrigação interfere nas trocas energéticas entre a comunidade vegetal e a atmosfera circundante, aumentando a relação LE/Sr e diminuindo H/Sr.

Desta maneira, conclui-se que a variação na partição do $\mathrm{Sr}$ foi condicionada pelo período em que se desenvolveu a pesquisa, o qual se caracterizou com grande variação na nebulosidade e chuvas intensas que contribuíram para que uma parcela maior do saldo de radiação fosse utilizada com fluxo de calor latente e em menores proporções, como fluxo de calor sensível e fluxo de calor no solo.

\section{Conclusões}

1. Não houve mudanças significativas no comportamento dos componentes do balanço de radiação.

2. O albedo apresentou valores decrescentes com o crescimento e desenvolvimento da cultura variando de 0,23 na fase de crescimento dos colmos a 0,21 na maturação enquanto o valor médio do albedo para todo o ciclo da cultura foi 0,23 .

3. O coeficiente térmico $(\tau)$ diminui com o crescimento da cultura ocorrendo o contrário com o coeficiente de transformação em ondas longas $(\lambda)$. 
4. O saldo de radiação diurno foi menor no período chuvo-so e máximo no período seco. Os fluxos de calor latente, calor sensível e calor no solo, seguiram aquele do saldo de radiação.

5. O saldo de radiação foi consumido em aproximadamente $82 \%$ como fluxo de calor latente, $16 \%$ como fluxo de calor sensível para aquecer o ar e $2 \%$ como fluxo de calor sensível para o solo.

\section{Agradecimentos}

Os autores agradecem à Miriri Alimentos e Bioenergia S/A, pelo apoio e concessão da área e ao $\mathrm{CNPq}$, pelo apoio financeiro e concessão de Bolsa de Mestrado.

\section{Literatura Citada}

André, R. G. B.; Mendonça, J. C.; Marques, V. da S.; Pinheiro, F. M. A.; Marques, J. Aspectos energéticos do desenvolvimento da cana-de-açúcar. Parte 1: Balanço de radiação e parâmetros derivados. Revista Brasileira de Meteorologia, v.25, p.375382, 2010a.

André, R. G. B.; Mendonça, J. C.; Pinheiro, F. M. A.; Marques, V. da S.; Marques, J. Aspectos energéticos do desenvolvimento da cana-de-açúcar. Parte 2: Balanço de energia e parâmetros derivados. Revista Brasileira de Meteorologia, v.25, p.535542, 2010b.

Armas, E. D.; Monteiro, R. T. R.; Amancio, A. V.; Correa, M. R. L.; Guercio, M. A. Uso de agrotóxicos em cana-de-açúcar na Bacia do Rio Corumbataí e o risco de poluição hídrica. Química Nova, v.28, p.975-982, 2005.

Azevedo, P. V. de; Silva, B. B. da; Silva, V. de P. R. da. Water requirements of irrigated mango orchards in Northeast Brazil. Agricultural Water Management, v.58, p.241-254, 2003.

Azevedo, P. V. de; Sousa, I. F.; Silva, B. B. da; Silva, V. de P. R. da. Water use efficiency of dwarf-green coconut (Cocos nucifera L.) orchards in Northeast Brazil. Agricultural Water Management, v.1, p.259-264, 2006.

Borges, C. J. R.; Azevedo, P. V. de; Silva, V. de P. R. da; Campos, J. H. B. C.; Moura, M. S. B.; Soares, J. M.; Silva, B. B. da. Influência do calor armazenado no sistema solo-planta no balanço de energia em pomar de mangueiras. Revista Brasileira de Engenharia Agrícola e Ambiental, v.12, p.393399, 2008.

Cabral, O. M. R.; Rocha, H. R.; Ligo, M. A. V.; Brunini, O.; DIAS, M. A. F. S. Fluxos turbulentos de calor sensível, vapor d'água e CO2 sobre plantação de cana-de-açúcar (Saccharum sp.) em Sertãozinho-SP. Revista Brasileira de Meteorologia, v.18, p.61-70, 2003.

Esteves, B. dos S.; Sousa, E. F. de; Mendonça, J. C.; Lousada, L. de L.; Muniz, R. de A.; Siqueira, D. P. Albedo da cana-de-açucar em Campos dos Goytacazes, RJ. In: Congresso Brasileiro de Agrometeorologia, 17, 2011, Guarapari. Anais...Guarapari: SBAGRO, 2011. CD-Rom
Gavilán, P.; Berengena, E. J. Accuracy of the Bowen ratioenergy balance method for measuring latent heat flux in a semiarid advective environment. Irrigation Science, v.25, p.127-140, 2007.

IBGE - Instituto Brasileiro de Geografia e Estatística. Estatística da produção agrícola março de 2012. http://www.ibge. gov.br/home/estatistica/indicadores/agropecuaria/lspa/ estProdAgr_201203.pdf. 17 Abr. 2013.

Inman-Bamber, N. G.; Mcglinchey, M. G. Crop coefficients and water-use estimates for sugarcane based on long-term bowen ratio energy balance measurements. Field Crops Research, v.83, p.125-138, 2003.

Kjaersgaard, J. H.; Plauborg, F.; Mollerup, M.; Petersen, C. T.; Hansen, S. Crop coefficients for winter wheat in a subhumid climate regime. Agricultural Water Management, v.95, p.918-924, 2008.

Oliveira, G. M. de; Leitão, M. V. B. R. Estimativas de evapotranspiração e as conseqüências devido aos erros na determinação de saldo de radiação e efeitos advectivos. Revista Brasileira de Engenharia Agrícola e Ambiental, v.4, p.343-347, 2000.

Ortega-Farias, S. O.; Cuenca, R. H.; Ek, M. Daytime variation of sensible heat flux estimated by the bulk aerodynamic method over a grass canopy. Agricultural and Forest Meteorology, v.81, p.131-143, 1996.

Perez, P. J.; Castellvi, F.; Ibañez, M.; Rosell, J. I. Assessment of reliability of Bowen ratio method for partitioning fluxes. Agricultural and Forest Meteorology, v.97, p.141150, 1999.

Perez, P. J.; Castellvi, F.; Martinez-cob, A. A simple model for estimating the Bowen ratio from climatic factors for determining latent and sensible heat flux. Agricultural Forest Meteorology, v.148, p.25-37, 2008.

Qin, Z.; Ouyang, Y.; Su, G.; Yu, Q.; Li, J.; Zhang, J.; Wu, Z. Characterization of $\mathrm{CO} 2$ and water vapor fluxes in a summer maize field with wavelet analysis. Ecological Informatics, v.3, p.397-409, 2008.

Righi, E. Z.; Angelocci, L. R.; Marin, F. R. Energy balance of a young drip-irrigated coffe crop in shoutheast Brazil: An analysis of errors and reliability of measurements by the Bowen ratio method. Revista Brasileira de Agrometeorologia, v.15, p.367-279, 2007.

Shen, Y.; Zhang, Y.; Kondoh, A.; Tang, C.; Chen, J.; Xias, J.; Sakllra, Y.; Liu, C.; Sun, H. Seasonal variation of energy partitioning in irrigated lands. Hydrological Processes, v.18, p.2223-2234, 2004.

Silva, B. B.; Lopes, G. M.; Azevedo, P. V. de. Balanço de radiação em áreas irrigadas utilizando imagens Landsat 5 - TM. Revista Brasileira de Meteorologia, v.20, 243-252, 2005.

Silva, T. G. F. da; Moura, M. S. B. de; Zolnier, S.; Soares, J. M.; Souza, L. S. B. de; Brandão, E. O. Variação do balanço de radiação e de energia da cana-de-açúcar irrigada no semiárido brasileiro. Revista Brasileira de Engenharia Agrícola e Ambiental, v.15, p.139-147, 2011.

Silva, V. de P. R. da. On climate variability in Northeast of Brazil. Journal of Arids Environments, v.58, p.575-596,2004. 
Silva, V. de P. R. da; Campos, J. H. B. C.; Azevedo, P. V. de. Water-use efficiency and evapotranspiration of mango orchard grown in northeastern region of Brazil. Scientia Horticulturae, v.102, p.467-472, 2009.

Teixeira, A. H. de C. Avaliação dos componentes do balanço de energia durante o primeiro ano de cultura da banana. Revista Brasileira Engenharia Agrícola e Ambiental, v.5, p.28-32, 2001.
Teixeira, A. H. de C.; Bastiaanssen, W. G. M.; Ahmad, N. D.; Moura, M. S. B.; Bos, M. G. Analysis of energy fluxes and vegetation-atmosphere parameters in irrigated and natural ecosystems of semi-arid Brazil. Journal of Hydrology, v.362, p.110-127, 2008.

Vianello, R. L.; Alves, A. R. Meteorologia básica e aplicações. Viçosa: Imprensa Universitária, UFV, 1991. 449p. 